\title{
EDITORIAL
}

\section{Standardised methodology of sputum induction and processing}

\author{
R. Djukanović*, P.J. Sterk", J.V. Fahy ${ }^{\text {I,+}}$, F.E. Hargreave ${ }^{\S}$
}

Research into the pathogenetic mechanisms and clinical monitoring of inflammatory diseases of any system is complete only if it involves the study of both the underlying pathological features and the physiological consequences that result from what are always complex inflammatory processes. Respiratory diseases, including those of the airways, are no exception in this respect. It is, therefore, not surprising that the development over the last 25 years of techniques enabling the study of inflammatory processes in the airways has revolutionised understanding of the commonest pulmonary diseases, asthma and chronic obstructive pulmonary disease (COPD). Airways inflammation is now an established feature and a central consideration of any treatment strategy for both of these conditions.

Most of the initial observations made in asthma, documenting the involvement of eosinophils, mast cells, T-cells and more recently structural cells, fibroblasts, endothelial cells and epithelial cells, were made in studies using fibreoptic bronchoscopy in conjunction with bronchoalveolar lavage and bronchial biopsy. However, the relative invasiveness of this technique restricted the use of bronchoscopy to a research setting that was available in a limited number of specialised centres. This did not allow bronchoscopy to develop into a practical diagnostic tool which would guide the clinician in routine practice. Similarly, it was not easy to apply bronchoscopy in large clinical trials.

The ability to study inflammation has changed considerably with the development of the induced sputum technique as a research and, increasingly, clinical tool. Although it has been known for decades that sputum produced spontaneously by asthmatic individuals contains numerous eosinophils, it is only recently that attempts have been made to validate and standardise the use of sputum induced using hypertonic saline as a tool to study inflammatory events in the lungs. Since the first description of a standardised method in 1992 by PIN et al. [1], there has been an impressive increase in the number of papers in which researchers have used induced sputum to study various aspects of airways inflammation, focusing initially on bronchial asthma and extending the research more recently into COPD.

For any technique to be used widely and for observations between centres to be comparable between different research groups, it needs to be validated and standardised. The first attempt to do this for the induced sputum technique was made at a workshop which took place in 1996 in Stockholm, Sweden. A number of issues, including standardisation and analysis of samples, were raised at this meeting. Some of these were followed through by individual groups. However, over the course of several subsequent meetings, it became evident that only a concerted effort would resolve some of the most important issues such as the optimum method for inducing sputum, its homogenisation and analysis of the large number of components.

The initiative to organise the second formal workshop involving all the major contributors in the field of sputum induction finally matured by the time of the European Respiratory Society (ERS) meeting in Berlin, Germany, in 1997. It was agreed that the first in a series of workshops would be held in Geneva, Switzerland, in 1998, just prior to the ERS meeting, at which four aspects of sputum induction would be discussed: 1) sputum induction and processing, 2) read-outs from sputum (inflammatory markers), 3) the value of induced sputum in clinical trials, and 4) its value in clinical practice.

After this meeting, which was very successful, it was decided that a Task Force would be set up under the auspices of the ERS. Aims and objectives were identified and working groups established to deal with individual aspects of sputum induction and its safety, processing for cell and fluid-phase analysis, clinical application, use in the study of airway inflammation in children and use in clinical trials. A final group whose aim was to look at the future of the induced sputum technique was also set up. The Task Force was approved by the ERS in September 1999. The Task Force members set to work; meetings took place at the ERS Conference in Madrid, Spain, the American Thoracic Society (ATS) meeting in Toronto, Canada, and finally in Florence, Italy, in 2000. At the meetings, the eight working groups met

\footnotetext{
*Southampton University General Hospital, Southampton, UK. ${ }^{\#}$ Leiden University Medical Centre, Leiden, the Netherlands. ${ }^{\text {Pulmonary }}$ and Critical Care Division, Dept of Medicine, and Cardiovascular Research Institute, University of California at San Francisco, San Francisco, CA, USA. ${ }^{\S}$ Asthma Research Group, Firestone Institute for Respiratory Health, McMaster University, Hamilton, Ontario, Canada.
}

Correspondence: R. Djukanović, Division of Infection, Inflammation and Repair, Respiratory Cell \& Molecular Biology, Mailpoint 810, Level D, Centre Block, Southampton General Hospital, Southampton, SO16 6YD, UK. Fax: 4423 80777996. E-mail: rd1@soton.ac.uk 
under the chairmanship of their working group leaders and discussed issues pertaining to their own subject. Meetings always ended with summaries presented by the working group leaders, allowing all Task Force members to comment. Between meetings, communication and writing up of the Task Force document continued. The final document was approved in Florence, although minor amendments were made subsequently at the ATS meeting in San Francisco, CA, USA. Then followed the painful process of editing this large document to ensure uniformity of style and referencing.

This report summarises the work and conclusions of the Task Force. It sums up the knowledge that has accumulated in the field of induced sputum and provides guidance on the most appropriate methods for induction and analysis of the cellular and soluble phases of sputum. The scope of this report is limited to the two main airway diseases, asthma and COPD, and does not include the use of induced sputum in the diagnosis and research of infectious diseases, interstitial lung diseases or cancer. The Task Force has identified outstanding issues which need to be addressed either by individual groups or as part of a combined effort. It is hoped that this document will provide helpful guidance to both researchers and clinical practitioners regarding the use of the induced sputum technique.

The co-chairmen of the Task Force would like to thank all the members who contributed to this huge undertaking.

\section{References}

1. Pin I, Gibson PG, Kolendowicz R, et al. Use of induced sputum cell counts to investigate airway inflammation in asthma. Thorax 1992; 47: 25-29. 